\title{
Spherical spectral synthesis
}

\author{
László Székelyhidi \\ Institute of Mathematics, University of Debrecen, \\ e-mail: 1szekelyhidi@gmail.com
}

\begin{abstract}
In this paper we make an attempt to extend L. Schwartz's classical result on spectral synthesis to several dimensions. Due to counterexamples of D. I. Gurevich this is impossible for translation invariant varieties. Our idea is to replace translations by proper euclidean motions in higher dimensions. For this purpose we introduce the basic concepts of spectral analysis and synthesis in the non-commutative setting based on Gelfand pairs, where "translation invariance" will be replaced by invariance with respect to a compact group of automorphisms. The role of exponential functions will be played by spherical functions. As an application we obtain the extension of L. Schwartz's fundamental result.
\end{abstract}

\section{Notation and terminology}

In this paper we propose a general setting for spectral analysis and synthesis on non-commutative locally compact groups. It is clear that exponentials and exponential monomials, which serve as basic building blocks for spectral synthesis on commutative groups will not be able to play a similar role on noncommutative groups. Nevertheless, some commutative ideas can be utilized in the case of Gelfand pairs. In our approach exponentials will be replaced by spherical functions and translation invariance will be replaced by invariance with respect to a given compact subgroup, in particular, to a compact group of automorphisms. We apply these ideas in the case of the Gelfand pair $\left(\mathbb{R}^{n}, S O(n)\right)$, where the classical concept of "translation invariance" in spectral synthesis will be replaced by invariance under Euclidean motions. It turns out that this "spherical spectral synthesis" can be considered as a generalization of L. Schwartz's classical spectral synthesis result from the reals to $\mathbb{R}^{n}$.

Although the basics of the theory of spherical functions and Gelfand pairs can be found in [1] (see also 2]) here we include all necessary concepts and results - for the sake of completeness.

The research was partly supported by the Hungarian National Foundation for Scientific Research (OTKA), Grant No. K111651.

Keywords and phrases: Gelfand pair, spherical function, spherical monomial, spectral synthesis

AMS (2000) Subject Classification: 43A45, 43A90, 22D15 
In this paper $\mathbb{C}$ denotes the set of complex numbers. For a locally compact group $G$ we denote by $\mathcal{C}(G)$ the locally convex topological vector space of all continuous complex valued functions defined on $G$, equipped with the pointwise operations and with the topology of uniform convergence on compact sets. For each function $f$ in $\mathcal{C}(G)$ we define $\breve{f}$ by $\breve{f}(x)=f\left(x^{-1}\right)$, whenever $x$ is in $G$.

It is known that the dual of $\mathcal{C}(G)$ can be identified with the space $\mathcal{M}_{c}(G)$ of all compactly supported complex Borel measures on $G$ which is equipped with the pointwise operations and with the weak*-topology. The pairing between $\mathcal{C}(G)$ and $\mathcal{M}_{c}(G)$ is given by the formula

$$
\langle\mu, f\rangle=\int f d \mu
$$

We shall use the following theorem, describing the dual of $\mathcal{M}_{c}(G)$. A more general theorem together with the proof can be found in [4, 17.6, p. 155.

Theorem 1. Let $G$ be a locally compact group. For every weak*-continuous linear functional $F: \mathcal{M}_{c}(G) \rightarrow \mathbb{C}$ there exists a unique continuous function $f: G \rightarrow \mathbb{C}$ such that $F(\mu)=\mu(f)$ for each $\mu$ in $\mathcal{M}_{c}(G)$.

The measure $\breve{\mu}$ is defined by

$$
\langle\breve{\mu}, f\rangle=\langle\mu, \breve{f}\rangle
$$

for each $\mu$ in $\mathcal{M}_{c}(G)$ and $f$ in $\mathcal{C}(G)$.

Convolution in $\mathcal{M}_{c}(G)$ is defined by

$$
\langle\mu * \nu, f\rangle=\int f(x y) d \mu(x) d \nu(y)
$$

for each $\mu, \nu$ in $\mathcal{M}_{c}(G)$ and $x$ in $G$. Convolution converts the linear space $\mathcal{M}_{c}(G)$ into a topological algebra with unit $\delta_{e}, e$ being the identity in $G$.

Convolution of measures in $\mathcal{M}_{c}(G)$ with arbitrary functions in $\mathcal{C}(G)$ is defined by the similar formula

$$
\mu * f(x)=\int f\left(y^{-1} x\right) d \mu(y)
$$

for each $\mu$ in $\mathcal{M}_{c}(G), f$ in $\mathcal{C}(G)$ and $x$ in $G$. It is clear that, equipped with the action $f \mapsto \mu * f$, the space $\mathcal{C}(G)$ is a topological left module over $\mathcal{M}_{c}(G)$.

In what follows $K$ will always denote a compact subgroup in $G$ with normed Haar measure $\omega$. We recall that $\omega$ is left invariant, right invariant and inversion invariant.

The function $f$ in $\mathcal{C}(G)$ is called bi-K-invariant, or simply $K$-invariant if it satisfies

$$
f(k x l)=f(x)
$$


for each $x$ in $G$ and $k, l$ in $K$. All $K$-invariant functions form a closed subspace in the topological vector space $\mathcal{C}(G)$, which we denote by $\mathcal{C}(G / / K)$. Clearly, $\breve{f}$ is $K$-invariant, if $f$ is $K$-invariant.

For each $f$ in $\mathcal{C}(G)$ the function defined by

$$
f^{\#}(x)=\int_{K} \int_{K} f(k x l) d \omega(k) d \omega(l),
$$

for $x$ in $G$ is called the projection of $f$. Obviously, $f^{\#}$ is $K$-invariant, further the function $f$ in $\mathcal{C}(G)$ is $K$-invariant if and only if $f^{\#}=f$. We note that if $G$ is Abelian, then the space $\mathcal{C}(G / / K)$ can be identified with $\mathcal{C}(G / K)$. Clearly, $\left(f^{\#}\right)^{\llcorner}=(\breve{f})^{\#}$ holds for each $f$ in $\mathcal{C}(G)$.

A measure $\mu$ in $\mathcal{M}_{c}(G)$ is called $K$-invariant, if

$$
\langle\mu, f\rangle=\left\langle\mu, f^{\#}\right\rangle
$$

holds for each $f$ in $\mathcal{C}(G)$. We also define the projection $\mu^{\#}$ of $\mu$ by the equation

$$
\left\langle\mu^{\#}, f\right\rangle=\int_{G} \int_{K} \int_{K} f(k x l) d \omega(k) d \omega(l) d \mu(x)
$$

for each $f$ in $\mathcal{C}(G)$. Obviously, $\mu^{\#}$ is in $\mathcal{M}_{c}(G)$ and it is $K$-invariant, further $\left\langle\mu^{\#}, f\right\rangle=\left\langle\mu, f^{\#}\right\rangle$. It follows that the measure $\mu$ in $\mathcal{M}_{c}(G)$ is $K$-invariant if and only if $\mu^{\#}=\mu$.

A special role is played by the projections of the evaluation functionals $\delta_{y}$ defined by

$$
\left\langle\delta_{y}, f\right\rangle=f(y)
$$

for each $y$ in $G$ and $f$ in $\mathcal{C}(G)$, hence

$$
\left\langle\delta_{y}^{\#}, f\right\rangle=f^{\#}(y)=\int_{K} f(k y l) d \omega(k) d \omega(l) .
$$

Using these measures we define $K$-translation by $y$ in $G$ for each $f$ in $\mathcal{C}(G)$ as the function $\tau_{y} f$ defined by the equation

$$
\tau_{y} f(x)=\delta_{y^{-1}}^{\#} * f(x)=\int_{K} \int_{K} f(k y l x) d \omega(k) d \omega(l)
$$

for each $x$ in $G$. In particular, for each $K$-invariant function $f$ we have

$$
\tau_{y} f(x)=\int_{K} f(y k x) d \omega(k)
$$

whenever $x, y$ are in $G$. A subset $H$ in $\mathcal{C}(G / / K)$ is called $K$-translation invariant, if for each $f$ in $H$ and $y$ in $G$ the function $\tau_{y} f$ is in $H$. A closed $K$ translation invariant linear subspace of $\mathcal{C}(G / / K)$ is called a $K$-variety. Clearly, the intersection of any family of $K$-varieties is a $K$-variety. The intersection of all $K$-varieties including the $K$-invariant function $f$ is called the $K$-variety generated by $f$, or simply the $K$-variety of $f$, and it is denoted by $\tau(f)$. This is the closure of the linear space spanned by all $K$-translates of $f$. 


\section{The dual of $\mathcal{C}(G / / K)$}

The following theorem describes the space of all continuous linear functionals of the space $\mathcal{C}(G / / K)$, that is, the dual space $\mathcal{C}(G / / K)^{*}$.

Theorem 2. The dual of $\mathcal{C}(G / / K)$ is identical with the set of the restrictions of all $K$-invariant measures in $\mathcal{M}_{c}(G)$ to $\mathcal{C}(G / / K)$.

Proof. Suppose that $\lambda$ is in $\mathcal{C}(G / / K)^{*}$ and we define

$$
\langle\tilde{\lambda}, f\rangle=\left\langle\lambda, f^{\#}\right\rangle
$$

for each $f$ in $\mathcal{C}(G)$. Then obviously $\tilde{\lambda}$ is in $\mathcal{M}_{c}(G)$. We have

$$
\left\langle(\tilde{\lambda})^{\#}, f\right\rangle=\left\langle\tilde{\lambda}, f^{\#}\right\rangle=\left\langle\lambda, f^{\# \#}\right\rangle=\left\langle\lambda, f^{\#}\right\rangle=\langle\tilde{\lambda}, f\rangle
$$

for each $f$ in $\mathcal{C}(G)$, hence $\tilde{\lambda}$ is $K$-invariant. If $f$ is in $\mathcal{C}(G / / K)$, then $f=f^{\#}$ and we infer

$$
\langle\tilde{\lambda}, f\rangle=\left\langle\tilde{\lambda}, f^{\#}\right\rangle=\left\langle\lambda, f^{\# \#}\right\rangle=\left\langle\lambda, f^{\#}\right\rangle=\langle\lambda, f\rangle
$$

hence $\tilde{\lambda}$ coincides with $\lambda$ on $\mathcal{C}(G / / K)$. This shows that $\lambda$ is the restriction of a $K$-invariant measure in $\mathcal{M}_{c}(G)$.

Conversely, it is clear that the restriction of every $K$-invariant measure in $\mathcal{M}_{c}(G)$ to $\mathcal{C}(G / / K)$ is a linear functional on $\mathcal{C}(G / / K)$, hence it is an element of $\mathcal{C}(G / / K)^{*}$.

The dual of $\mathcal{C}(G / / K)$ will be denoted by $\mathcal{M}_{c}(G / / K)$. It follows from the previous theorem that with the convolution of measures restricted to $\mathcal{M}_{c}(G / / K)$ the latter is a topological algebra with unit $\delta_{e}$. Further, $\mathcal{C}(G / / K)$ is a left module over $\mathcal{M}_{c}(G / / K)$ with the ordinary convolution $f \mapsto \mu * f$. Closed submodules of this module are exactly the $K$-varieties. Left, right and two-sided ideals in $\mathcal{M}_{c}(G / / K)$ are called left $K$-ideal, right $K$-ideal and $K$-ideal, respectively.

Theorem 3. All finitely supported $K$-invariant measures form a dense subalgebra in $\mathcal{M}_{c}(G / / K)$.

Proof. Let $\mu$ be a $K$-invariant measure and let $\left(\nu_{\alpha}\right)_{\alpha \in A}$ be a generalized sequence of finitely supported measures in $\mathcal{M}_{c}(G)$ converging to $\mu$ in the weak*topology. Then $\left(\nu_{\alpha}^{\#}\right)_{\alpha \in A}$ is a generalized sequence of finitely supported measures in $\mathcal{M}_{c}(G / / K)$ converging to $\mu^{\#}=\mu$ in the weak*-topology.

\section{Orthogonal complements and annihilators}

Theorem 4. Let I be a left $K$-ideal. Then

$$
I^{\perp}=\{f: f \in \mathcal{C}(G / / K),\langle\mu, f\rangle=0 \text { for each } \mu \in I\}
$$

is a $K$-variety. 
Proof. Clearly, $I^{\perp}$ is a closed linear space in $\mathcal{C}(G / / K)$. Let $f$ be in $I^{\perp}$ and $y$ in $G$, then we have for each $\mu$ in $I$

$$
\begin{gathered}
\left\langle\mu, \delta_{y^{-1}}^{\#} * f\right\rangle=\int_{G} \int_{K} \int_{K} f(k y l x) d \omega(k) d \omega(l) d \mu(x)= \\
\int_{G} \int_{G} f(u x) d \delta_{y}^{\#}(u) d \mu(x)=\int_{G} f(t) d\left(\delta_{y}^{\#} * \mu\right)(t)=\left\langle\delta_{y}^{\#} * \mu, f\right\rangle=0,
\end{gathered}
$$

as $\delta_{y}^{\#} * \mu$ is in $I$.

Theorem 5. Let $V$ be a $K$-variety. Then

$$
V^{\perp}=\left\{\mu: \mu \in \mathcal{M}_{c}(G / / K),\langle\mu, f\rangle=0 \text { for each } f \in V\right\}
$$

is a closed left $K$-ideal.

Proof. Obviously, $V^{\perp}$ is a closed linear subspace in $\mathcal{M}_{c}(G / / K)$. Let $\mu$ in $V^{\perp}$, $\nu$ in $\mathcal{M}_{c}(G / / K)$, and $f$ in $V$. Then we have to show that

$$
\langle\nu * \mu, f\rangle=\int_{G} f(x y) d \nu(x) d \mu(y)=0 .
$$

As convolution in $\mathcal{M}_{c}(G / / K)$ is continuous in both variables, it is enough to prove this for $\nu=\delta_{u}^{\#}$, by Theorem [3, where $u$ is in $G$. On the other hand, we have, as above

$$
\left\langle\delta_{u}^{\#} * \mu, f\right\rangle=\left\langle\mu, \delta_{u^{-1}}^{\#} * f\right\rangle=0,
$$

by the $K$-translation invariance of $V$.

Obviously, the statement remains true if we assume only that $V$ is a $K$ translation invariant set.

Theorem 6. For each $K$-variety $V$ we have $V^{\perp \perp}=V$. For each closed left $K$-ideal $I$ we have $I^{\perp \perp}=I$.

Proof. Obviously, we have $V^{\perp \perp} \supseteq V$ and $I^{\perp \perp} \supseteq I$.

Suppose now that $V^{\perp \perp} \supsetneq V$. Consequently, there is a function $f$ in $V^{\perp \perp}$ such that $f$ is not in $V$. By the Hahn-Banach Theorem, and by Theorem 2 there is a $\lambda$ in $\mathcal{M}_{c}(G / / K)$ such that $\langle\lambda, f\rangle \neq 0$, and $\lambda$ vanishes on $V$. This means that $\lambda$ is in $V^{\perp}$, and $f$ is not in $V^{\perp \perp}$, a contradiction. Hence $V^{\perp \perp}=V$.

Similarly, suppose that $I^{\perp \perp} \supsetneq I$ and let $\mu$ be in $I^{\perp \perp}$ such that $\mu$ is not in $I$. As $I$ is closed, by the Hahn-Banach Theorem, there is a linear functional $\Lambda$ in the dual space $\mathcal{M}_{c}(G / / K)^{*}$ such that $\Lambda$ annihilates $I$ but $\Lambda(\mu) \neq 0$. By Theorem 1, every weak*-continuous linear functional on a dual space arises from an element of the original space, that is, there is an $f_{\Lambda}$ in $\mathcal{C}(G / / K)$ with $\Lambda(\nu)=\left\langle\nu, f_{\Lambda}\right\rangle$ holds for each for each $\nu$ in $\mathcal{M}_{c}(G / / K)$. As $\Lambda$ annihilates $I$ we have

$$
\Lambda(\nu)=\left\langle\nu, f_{\Lambda}\right\rangle=0
$$


for each $\nu$ in $I$, hence $f_{\Lambda}$ is in $I^{\perp}$. On the other hand,

$$
0 \neq \Lambda(\mu)=\left\langle\mu, f_{\Lambda}\right\rangle
$$

a contradiction, as $\mu$ is in $I^{\perp \perp}$. The proof is complete.

Besides orthogonal complements $V^{\perp}$ and $I^{\perp}$ we consider annihilators as follows. As $\mathcal{C}(G / / K)$ is a topological module over the algebra $\mathcal{M}_{c}(G / / K)$, the annihilators of subsets in $\mathcal{C}(G / / K)$, resp. in $\mathcal{M}_{c}(G / / K)$ have the usual meaning from module theory. Let $V$, resp. $I$ be a $K$-variety, resp. a left $K$-ideal. Then the annihilator of $V$ in $\mathcal{M}_{c}(G / / K)$, resp. of $I$ in $\mathcal{C}(G / / K)$ is defined as

$$
\text { Ann } V=\left\{\mu: \mu \in \mathcal{M}_{c}(G / / K) \text { and } \mu * f=0 \text { for each } f \text { in } V\right\} \text {, }
$$

resp.

$$
\text { Ann } I=\{f: f \in \mathcal{C}(G / / K) \text { and } \mu * f=0 \text { for each } \mu \text { in } I\} .
$$

Clearly, Ann $V$, resp. Ann $I$ are closed subspaces in $\mathcal{M}_{c}(G / / K)$, resp. in $\mathcal{C}(G / / K)$. We shall use the notation

$$
\breve{H}=\{\check{f}: f \in H\}, \breve{L}=\{\breve{\mu}: \mu \in L\} .
$$

for each subset $H$ in $\mathcal{C}(G)$ and $L$ in $\mathcal{M}_{c}(G)$.

Lemma 1. For each $K$-variety $V$ and left $K$-ideal $I$ we have

$$
\text { Ann } V=(\breve{V})^{\perp}, \quad \text { Ann } I=(\check{I})^{\perp} .
$$

Proof. Let $\mu$ be in Ann $V$ and $f$ in $V$, then we have

$$
\langle\mu, \check{f}\rangle=\int_{G} f\left(x^{-1}\right) d \mu(x)=\mu * f(e)=0,
$$

hence $\mu$ is in $(\breve{V})^{\perp}$. Conversely, let $\mu$ be in $(\breve{V})^{\perp}$ and $f$ in $V$. Then

$$
\begin{gathered}
\mu * f(x)=\mu^{\#} * f(x)=\int_{G} f\left(y^{-1} x\right) d \mu^{\#}(y)= \\
\int_{G} \int_{K} \int_{K} f\left(k y^{-1} l x\right) d \omega(k) d \omega(l) d \mu(y)=\int_{G} \int_{K} f\left(y^{-1} l x\right) d \omega(l) d \mu(y)= \\
\int_{G} \int_{K} \check{f}\left(x^{-1} l y\right) d \omega(l) d \mu(y)=\left\langle\mu, \delta_{x}^{\#} * \check{f}\right\rangle=0
\end{gathered}
$$

as $\breve{V}$ is $K$-variety, hence $\delta_{x}^{\#} * \check{f}$ is in $\breve{V}$.

To prove the second statement we suppose that $f$ is in $(\breve{I})^{\perp}$ and $\mu$ is in $I$. We can proceed as follows:

$$
\mu * f(x)=(\mu * f)^{\smile}\left(x^{-1}\right)=\int_{G}(\mu * f)^{\smile}(t) d \delta_{x^{-1}}(t)=
$$




$$
\begin{gathered}
\int_{G}\left[(\mu * f)^{\smile}\right]^{\#}(t) d \delta_{x^{-1}}(t)=\int_{G}(\mu * f)^{\smile}(t) d \delta_{x^{-1}}^{\#}(t)= \\
(\mu * f)\left(t^{-1}\right) d \delta_{x^{-1}}^{\#}(t)=\delta_{x^{-1}}^{\#} *(\mu * f)(e)=\left(\delta_{x^{-1}}^{\#} * \mu\right) * f(e)= \\
\left\langle\delta_{x^{-1}}^{\#} * \mu, \breve{f}\right\rangle=\left\langle\left(\delta_{x^{-1}}^{\#} * \mu\right)^{\smile}, f\right\rangle=0,
\end{gathered}
$$

as $\delta_{x^{-1}}^{\#} * \mu$ is in $I$. Conversely, if $f$ is in Ann $I$ and $\mu$ is in $\breve{I}$, then $\breve{\mu}$ is in $I$ and we infer

$$
0=\breve{\mu} * f(e)=\int_{G} f\left(t^{-1}\right) d \breve{\mu}(t)=\langle\mu, f\rangle,
$$

hence $f$ is in $(\breve{I})^{\perp}$.

Corollary 1. For each $K$-variety $V$ and closed $K$-ideal $I$ we have

$$
\operatorname{Ann}(\operatorname{Ann} V)=V, \quad \operatorname{Ann}(\operatorname{Ann} I)=I .
$$

\section{Gelfand pairs}

We call $(G, K)$ a Gelfand pair if the algebra $\mathcal{M}_{c}(G / / K)$ is commutative. Obviously, this is the case, for instance, if $G$ is commutative. We note that, although this definition is formally different from the one used in [1] (see also 2]) Theorem 2 and Theorem 9 below show that the two definitions coincide.

Theorem 7. $(G, K)$ is a Gelfand pair if and only if the measures $\left(\delta_{y}^{\#}\right)_{y \in G}$ form a commuting family.

Proof. The necessity is obvious, and the sufficiency follows from Theorem 3 and from the separate continuity of convolution in $\mathcal{M}_{c}(G / / K)$.

Let $\mathcal{C}_{c}(G)$ denote the space of compactly supported continuous complex valued functions on $G$ with the inductive limit topology of the subspaces of continuous complex valued functions with support in a given compact subset, equipped with the topology of uniform convergence. If $\lambda$ is a fixed left Haar measure on $G$, then with the convolution

$$
\varphi * \psi(x)=\int_{G} \varphi\left(t^{-1} x\right) \psi(t) d \lambda(t)
$$

$\mathcal{C}_{c}(G)$ is a topological algebra. For each $\varphi$ in $\mathcal{C}_{c}(G)$ we define

$$
\left\langle\mu_{\varphi}, f\right\rangle=\int_{G} f(x) \varphi(x) d \lambda(x)
$$

whenever $f$ is in $\mathcal{C}(G)$.

Theorem 8. The mapping $\varphi \mapsto \mu_{\varphi}$ is a continuous algebra homomorphism of $\mathcal{C}_{c}(G)$ into $\mathcal{M}_{c}(G)$ and its image is dense in $\mathcal{M}_{c}(G)$. 
Proof. Let $F(\varphi)=\mu_{\varphi}$ for each $\varphi$ in $\mathcal{C}_{c}(G)$. As the support of $\lambda$ is $G$, hence $\operatorname{supp} \mu_{\varphi}=\operatorname{supp} \varphi$. Then, clearly, the mapping $F: \mathcal{C}_{c}(G) \rightarrow \mathcal{M}_{c}(G)$ is linear. On the other hand, if $\left(\varphi_{\alpha}\right)_{\alpha \in A}$ converges to $\varphi$ in $\mathcal{C}_{c}(G)$, then let $L$ be a compact set in $G$ such that all the supports of the $\varphi_{\alpha}$ 's are in $L$. As $\lim _{\alpha}\left\|\varphi_{\alpha}-\varphi\right\|_{\infty} \rightarrow 0$ we have for each $f$ in $\mathcal{C}(G)$

$$
\begin{gathered}
\left|\left\langle F\left(\varphi_{\alpha}\right), f\right\rangle-\langle F(\varphi), f\rangle\right| \leqslant \int_{L}\left|\varphi_{\alpha}(x)-\varphi(x)\right||f(x)| d \lambda(x) \leqslant \\
\left\|\varphi_{\alpha}-\varphi\right\|_{\infty} \int_{L}|f(x)| d \lambda(x) \rightarrow 0,
\end{gathered}
$$

which proves the continuity of $F$. Let $\varphi, \psi$ be in $\mathcal{C}_{c}(G)$, then we have for each $f$ in $\mathcal{C}(G)$

$$
\begin{gathered}
\left\langle F\left(\varphi_{\alpha}\right) * F\left(\psi_{\alpha}\right), f\right\rangle=\left\langle\mu_{\varphi} * \nu_{\varphi}, f\right\rangle=\int_{G} f(x y) \varphi(x) \psi(y) d \lambda(x) d \lambda(y)= \\
\int_{G} f(z)\left[\int_{G} \varphi(x) \psi\left(x^{-1} z\right) d \lambda(x)\right] d \lambda(z)=\left\langle\mu_{\varphi_{\alpha} * \psi_{\alpha}}, f\right\rangle=\left\langle F\left(\varphi_{\alpha} * \psi_{\alpha}\right), f\right\rangle,
\end{gathered}
$$

hence $F$ is an algebra homomorphism. Finally, the last statement follows by regularization.

Corollary 2. Suppose that $G$ is unimodular. Then the restriction of the mapping $\varphi \rightarrow \mu_{\varphi}$ to $\mathcal{C}_{c}(G / / K)$ maps $\mathcal{C}_{c}(G / / K)$ into $\mathcal{M}_{c}(G / / K)$ and its image is dense in $\mathcal{M}_{c}(G / / K)$.

Proof. We have to show only that if $\varphi$ is $K$-invariant, then $\mu_{\varphi}$ is $K$-invariant, too. Using the notation $F$ from the previous theorem we can write for each $f$ in $\mathcal{C}(G)$ and for each $k, l$ in $K$

$$
\left\langle\mu_{\varphi}, f\right\rangle=\int_{G} f(x) \varphi(x) d \lambda(x)=\int_{G} f(k x l) \varphi(k x l) d \lambda(x)=\int_{G} f(k x l) \varphi(x) d \lambda(x) .
$$

Integrating over $K \times K$ we have

$$
\begin{gathered}
\left\langle\mu_{\varphi}, f\right\rangle=\int_{G} \int_{K} \int_{K} f(k x l) \varphi(x) d \omega(k) d \omega(l) d \lambda(x) \\
=\int_{G} f^{\#}(x) \varphi(x) d \lambda(x)=\left\langle\mu_{\varphi}, f^{\#}\right\rangle=\left\langle\mu_{\varphi}^{\#}, f\right\rangle,
\end{gathered}
$$

that is, $\mu_{\varphi}^{\#}=\mu_{\varphi}$, hence $\mu_{\varphi}$ is $K$-invariant.

Let $\mu^{\#}$ be in $\mathcal{M}_{c}(G / / K)$ and $\left(\varphi_{\alpha}\right)_{\alpha \in A}$ in $\mathcal{C}_{c}(G)$ such that

$$
\lim _{\alpha} \mu_{\varphi_{\alpha}}=\mu \text {. }
$$

Then obviously

$$
\lim _{\alpha} \mu_{\varphi_{\alpha}}^{\#}=\lim _{\alpha} \mu_{\varphi_{\alpha}^{\#}}=\mu^{\#}
$$

and the proof is complete. 
Theorem 9. Let $G$ be unimodular. Then $(G, K)$ is a Gelfand pair if and only if the convolution algebra $\mathcal{C}_{c}(G / / K)$ is commutative.

Proof. The necessity is obvious and the sufficiency is a consequence of the previous theorem.

Using the fact that $\mathcal{C}^{\infty}$ functions form a dense subset in $\mathcal{C}_{c}(G)$ with respect to the sup norm, we have the following corollary.

Corollary 3. Suppose that $G$ is unimodular. Then the restriction of the mapping $\varphi \rightarrow \mu_{\varphi}$ to $\mathcal{C}_{c}(G / / K) \cap \mathcal{C}^{\infty}(G)$ has a dense image in $\mathcal{M}_{c}(G / / K)$.

For the proofs of the following results see e.g. 2].

Theorem 10. (C. Berg) If $(G, K)$ is a Gelfand pair, then $G$ is unimodular.

Theorem 11. Suppose that there exists a continuous involutive automorphism $\theta: G \rightarrow G$ such that $\theta(x)$ is in $K x^{-1} K$ holds for each $x$ in $G$. Then $(G, K)$ is a Gelfand pair.

Corollary 4. Suppose that there exists a continuous involutive automorphism $\theta: G \rightarrow G$ such that the subgroup of all $\theta$-fixed points $K$ is compact, and every element $x$ of $G$ can be written in the form $x=k y$ with $k$ in $K$ and $\theta(y)=y^{-1}$. Then $(G, K)$ is a Gelfand pair.

Proof. Let $x=k y$ with $k$ in $K$ and $\theta(y)=y^{-1}$, then

$$
\theta(x)=\theta(k) \cdot \theta(y)=k \cdot y^{-1}=k \cdot x^{-1} \cdot k \in K x^{-1} K,
$$

hence the statement follows from the previous theorem.

\section{Semidirect products}

Let $N$ be a locally compact group and $K$ a compact topological group of continuous automorphisms of $N$. Hence, as a group, $K$ is a subgroup of the group $\operatorname{Aut}(N)$ of all continuous automorphisms of $N$, and $K$ is equipped with a compact topology which is compatible with the group structure, that is, $k \mapsto$ $k(n)$ is continuous on $K$ for each $n$ in $N$. We consider the semidirect product $G=K \ltimes N$, where the operation is defined by

$$
(k, n) \cdot(l, m)=(k \cdot l, k(m) \cdot n)
$$

for each $(k, n),(l, m)$ in $G$. The identity of this group is $e=(i d, e)$, where $i d$ is the identity mapping on $N$ and $e$ is the identity element of $N$, further, the inverse of $(k, n)$ is

$$
(k, n)^{-1}=\left(k^{-1}, k^{-1}\left(n^{-1}\right)\right) .
$$

In general, $K$ is isomorphic to the compact subgroup $\{(k, e): k \in K\}$ of $G$, and $N$ is isomorphic to the normal subgroup $\{i d, n): n \in N\}$. 
Suppose now that $N$ is commutative and we write the operation in $N$ as addition with $e=o$. If we define $\theta: G \rightarrow G$ by

$$
\theta(k, n)=(k,-n)
$$

for each $k$ in $K$ and $n$ in $N$, then we have that

$$
\begin{gathered}
\theta[(k, n)(l, m)]=\theta(k l, k(m)+n)=(k l,-k(m)-n)= \\
(k,-n)(l,-m)=\theta(k, n) \theta(l, m),
\end{gathered}
$$

that is, $\theta$ is a continuous involutive automorphism of $G$. On the other hand, we have

$$
\theta(k, n)=(k,-n)=(k, o)\left(k^{-1}, k^{-1}(-n)\right)(k, o)=(k, o)(k, n)^{-1}(k, o),
$$

that is, $\theta(k, n)$ is in $K \cdot(k, n)^{-1} \cdot K$ for each $(k, n)$ in $G$. By Theorem 11] we have the following result.

Corollary 5. With the above notation $G=K \ltimes N$ we have that $(G, K)$ is a Gelfand pair.

In this case we can say - somewhat loosely - that $(N, K)$ is a Gelfand pair. The continuous function $f: K \times N \rightarrow \mathbb{C}$ is $K$-invariant if and only if

$$
f(k, n)=f\left(\left(k^{\prime}, o\right)(k, n)\left(l^{\prime}, o\right)\right)=f\left(k^{\prime} k l^{\prime}, k^{\prime}(n)\right)
$$

for each $k, k^{\prime}, l^{\prime}$ in $K$ and $x$ in $\mathbb{N}$. With the choice $k^{\prime}=l$ and $l^{\prime}=k^{-1} l^{-1}$ it follows that $f(k, n)=f(i d, l(n))$ for each $k, l$ in $K$ and $n$ in $N$. This means that $K$-invariant functions depend on the second variable only, that is, they can be identified with continuous functions on $N$ by restriction $\left.f \mapsto f\right|_{N}$, and this restriction is invariant with respect to the action of $K$ on $N:\left.f\right|_{N}(k(n))=\left.f\right|_{N}(n)$ for each $k$ in $K$ and $n$ in $N$. These functions on $N$ are called $K$-radial functions and $\mathcal{C}(G / / K)$ will be identified with the space $\mathcal{C}_{K}(N)$ of all $K$-radial functions. Hence $f$ is in $\mathcal{C}_{K}(N)$ if and only if $f: N \rightarrow \mathbb{C}$ is a continuous function satisfying $f(k \cdot n)=f(n)$ for each $k$ in $K$ and $n$ in $N$. The dual $\mathcal{M}_{c}(G / / K)$ is the space of $K$-radial measures $\mu$ on $G$ satisfying

$$
\langle\mu, f\rangle=\int_{G} \int_{K} \int_{K} f\left(k^{\prime} k l^{\prime}, k^{\prime}(n)\right) d \omega\left(k^{\prime}\right) d \omega\left(l^{\prime}\right) d \mu(k, n)
$$

for each continuous function $f: K \times N \rightarrow \mathbb{C}$. Clearly, $K$-radial measures can be identified with those measures $\mu$ on $N$, which satisfy

$$
\int_{N} f(k(n)) d \mu(n)=\int_{N} f(n) d \mu(n)
$$

for each continuous function $f: N \rightarrow \mathbb{C}$ and for every $k$ in $K$. The space of $K$-radial measures will be denoted by $\mathcal{M}_{K}(N)$. 
Given a continuous function $f: K \times N \rightarrow \mathbb{C}$ its $K$-projection is the function

$$
f^{\#}(n)=\int_{K} f\left(k k^{\prime} l^{\prime}, k(n)\right) d \omega(k) d \omega\left(l^{\prime}\right)=\int_{K} f(k, k(n)) d \omega(k) .
$$

For each $(k, m)$ in $K \times N$ the $K$-radial measure $\delta_{(k, m)}^{\#}$ is independent of $k$ : $\delta_{(k, m)}^{\#}=\delta_{m}^{\#}$, and for each $K$-radial function $f$ we have

$$
\tau_{m} f(n)=\delta_{-m}^{\#} * f(n)=\int_{K} f(n+k(m)) d \omega(k)
$$

We obtain important special cases with the choice $N=\mathbb{R}^{n}$ and $K=O(n)$, or $K=S O(n)$, or $N=\mathbb{C}^{n}$ and $K=U(n)$, or $K=S U(n)$. In all these cases $\left(\mathbb{R}^{n}, K\right)$ is a Gelfand pair, and the space $\mathcal{C}(G / / K)$, resp. $\mathcal{M}_{c}(G / / K)$ will be identified with $\mathcal{C}_{K}\left(\mathbb{R}^{n}\right)$, resp. $\mathcal{M}_{K}\left(\mathbb{R}^{n}\right)$. Convolution in $\mathcal{M}_{K}\left(\mathbb{R}^{n}\right)$, resp. between $\mathcal{M}_{K}\left(\mathbb{R}^{n}\right)$ and $\mathcal{C}_{K}\left(\mathbb{R}^{n}\right)$ is the same as in $\mathcal{M}_{c}\left(\mathbb{R}^{n}\right)$, resp. between $\mathcal{M}_{c}\left(\mathbb{R}^{n}\right)$ and $\mathcal{C}\left(\mathbb{R}^{n}\right)$.

\section{Spherical functions}

From now on we suppose that $(G, K)$ is a Gelfand pair. For every $f$ in $\mathcal{C}(G / / K)$ and for each $y$ in $G$ the $K$-invariant measure

$$
D_{f ; y}=\delta_{y^{-1}}^{\#}-f(y) \delta_{e}
$$

is called the modified $K$-spherical difference corresponding to $f$ and $y$ (see [8, 9 , [10]). Given $f$ in $\mathcal{C}(G / / K)$ the closure of the $K$-ideal generated by all modified $K$-spherical differences of the form $D_{f ; y}$ with $y$ in $G$ will be denoted by $M_{f}$.

Theorem 12. Let $f$ be in $\mathcal{C}(G / / K)$. The $K$-ideal $M_{f}$ is proper if and only if $f(e)=1$, and $f$ satisfies

$$
\int_{K} f(x k y) d \omega(k)=f(x) f(y)
$$

for each $x, y$ in $G$. In this case $M_{f}$ is a maximal ideal and we have $\mathcal{M}_{c}(G / / K) / M_{f} \cong \mathbb{C}$.

Proof. Suppose that $M_{f}$ is a proper ideal. Then $V=M_{f}^{\perp}$ is a nonzero $K$ variety, by Theorems 4 and 6 . Let $\varphi \neq 0$ be in $V$, then we have for each $x, y, z$ in $G$

$$
\begin{gathered}
0=\left\langle D_{f ; y}, \delta_{z}^{\#} * \varphi\right\rangle=\left\langle\delta_{y^{-1}}^{\#}, \delta_{z}^{\#} * \varphi\right\rangle-f(y) \delta_{z}^{\#} * \varphi(e)= \\
\left(\delta_{z}^{\#} * \varphi\right)^{\#}\left(y^{-1}\right)-f(y) \int_{K} \int_{K} \varphi\left(k z^{-1} l\right) d \omega(k) d \omega(l)= \\
\int_{K} \int_{K} \int_{K} \varphi\left(z^{-1} l l^{\prime} y^{-1} k^{\prime}\right) d \omega(l) d \omega\left(l^{\prime}\right) d \omega\left(k^{\prime}\right)-f(y) \int_{K} \int_{K} \varphi\left(k z^{-1} l\right) d \omega(k) d \omega(l)=
\end{gathered}
$$




$$
\begin{gathered}
=\int_{K} \varphi\left(z^{-1} l y^{-1}\right) d \omega(l)-f(y) \int_{K} \varphi\left(z^{-1} l\right) d \omega(l)= \\
\int_{K} \varphi\left(z^{-1} l y^{-1}\right) d \omega(l)-f(y) \varphi\left(z^{-1}\right) .
\end{gathered}
$$

The substitution $z=e$ gives $\varphi\left(y^{-1}\right)=f(y) \varphi(e)$ thus $\varphi(e) \neq 0$ and we obtain equation (2) and $f(e)=1$.

We have proved that $V$ consists of all constant multiples of $\breve{f}$. In particular, $V$ is a one dimensional vector space, which implies, by Theorem 6 , that $M_{f}$ is a maximal ideal. We show that $\mathcal{M}_{c}(G / / K) / M_{f}$, as an algebra, is isomorphic to the algebra of complex numbers.

For each $\mu$ in $\mathcal{M}_{c}(G / / K)$ we define

$$
\Phi(\mu)=\langle\breve{\mu}, f\rangle .
$$

Clearly, $\Phi: \mathcal{M}_{c}(G / / K) \rightarrow \mathbb{C}$ is a surjective continuous linear functional. For $\mu, \nu$ in $\mathcal{M}_{c}(G / / K)$ we have

$$
\begin{gathered}
\Phi(\mu * \nu)=\langle\mu * \nu, \breve{f}\rangle=\int_{G} \int_{G} \breve{f}(u v) d \mu(u) d \nu(v)=\int_{G} \int_{G} \breve{f}(u v) d \mu^{\#}(u) d \nu^{\#}(v)= \\
\int_{G} \int_{G} \int_{K} \int_{K} \int_{K} \int_{K} \breve{f}\left(k u l l^{\prime} v k^{\prime}\right) d \omega(k) d \omega(l) d \omega\left(l^{\prime}\right) d \omega\left(k^{\prime}\right) d \mu(u) d \nu(v)= \\
\int_{G} \int_{G} \int_{K} \check{f}(u l v) d \omega(l) d \mu(u) d \nu(v)=\int_{G} \int_{G} \int_{K} f(u l v) d \omega(l) d \breve{\mu}(u) d \check{\nu}(v)= \\
\int_{G} \int_{G} f(u) f(v) d \breve{\mu}(u) d \breve{\nu}(v)=\Phi(\mu) \Phi(\nu),
\end{gathered}
$$

that is, $\Phi$ is an algebra homomorphism. On the other hand,

$$
\begin{gathered}
\Phi\left(D_{f ; y}\right)=\Phi\left(\delta_{y^{-1}}^{\#}\right)-f(y)= \\
\left\langle\left(\delta_{y^{-1}}^{\#}\right)^{\check{L}}, f\right\rangle-f(y)=\left\langle\delta_{y}^{\#}, f\right\rangle-f(y)=f^{\#}(y)-f(y)=0,
\end{gathered}
$$

as $f$ is $K$-invariant. It follows that $M_{f}$ is a subset of the kernel of $\Phi$. As $\Phi$ is continuous, its kernel is a closed maximal ideal, hence, in fact, $M_{f}$ is the kernel of $\Phi$. As $\Phi$ is surjective, we have $\mathcal{M}_{c}(G / / K) / M_{f} \cong \mathbb{C}$.

Now we suppose that $f(e)=1$, and $f$ satisfies (2). Then it is easy to check that $\breve{f}$ is in $M_{f}^{\perp}$, hence $M_{f}$ is proper, by Theorem 6 . The theorem is proved.

We call the nonzero $K$-invariant function $f$ a $K$-spherical function, if it satisfies equation (2) for each $x, y$ in $G$. In this case $f(e)=1$. By the previous theorem, there is a one-to-one correspondence between $K$-spherical functions on $G$ and those maximal ideals of the algebra $\mathcal{M}_{c}(G / / K)$ whose residual algebra is topologically isomorphic to $\mathbb{C}$. Such maximal ideals are called - in accordance with the terminology in the commutative case - exponential maximal ideals (see e.g. 8]). In other words, a maximal ideal in $\mathcal{M}_{c}(G / / K)$ is exponential, if it 
is the kernel of a continuous algebra homomorphism of $\mathcal{M}_{c}(G / / K)$ onto $\mathbb{C}$. In particular, every exponential maximal ideal is closed. It follows immediately that, in the case of commutative $G$, the $K$-spherical functions are exactly the exponentials of the group $G / K$.

We have the following characterization of $K$-spherical functions. We call the function $f$ in $\mathcal{C}(G)$ normed if $f(e)=1$.

Theorem 13. The $f$ be a continuous $K$-invariant function. Then the following statements are equivalent:

i) The function $f$ is a $K$-spherical function.

ii) The function $f$ is nonzero and satisfies (2) for each $x, y$ in $G$.

iii) The function $f$ is normed and for each $K$-invariant measure $\mu$ there exists a complex number $\lambda_{\mu}$ such that $\mu * f=\lambda_{\mu} \cdot f$

iv) The function $f$ is a common normed eigenfunction of all translation operators $\tau_{y}$ with $y$ in $G$.

v) The function $f$ is normed and the ideal $M_{f}$ is an exponential maximal ideal.

vi) The function $f$ is normed and the mapping $\mu \mapsto\langle\mu, \breve{f}\rangle$ is a nonzero multiplicative functional of the algebra $\mathcal{M}_{c}(G / / K)$ with kernel $M_{f}$.

Proof. The first two statements are equivalent, by definition.

Suppose that $f$ is nonzero and satisfies (2) for each $x, y$ in $G$. Let $\mu$ be a $K$-invariant measure, then we have

$$
\begin{gathered}
\mu * f(x)=\int_{G} f\left(y^{-1} x\right) d \mu(y)=\int_{G} \check{f}\left(x^{-1} y\right) d \mu(y)=\int_{G} \breve{f}\left(x^{-1} y\right) d \mu^{\#}(y)= \\
\int_{G} \int_{K} \int_{K} \check{f}\left(x^{-1} k y l\right) d \omega(k) d \omega(l) d \mu(y)=\int_{G} \int_{K} \check{f}\left(x^{-1} k y\right) d \omega(k) d \mu(y)= \\
\int_{G} \int_{K} f\left(y^{-1} k x\right) d \omega(k) d \mu(y)=\int_{G} f\left(y^{-1}\right) d \mu(y) \cdot f(x),
\end{gathered}
$$

which proves $i i i)$ with $\lambda_{\mu}=\int_{G} f\left(y^{-1}\right) d \mu(y)$.

As $\tau_{y} f=\delta_{y^{-1}} * f$, iii) obviously implies $i$ ).

If $f$ is a common normed eigenfunction of all translation operators $\tau_{y}$ with $y$ in $G$, then $\tau(f)$ is a one dimensional variety. It is easy to see that $\tau(\tilde{f})$ is a one dimensional variety, too. Hence $\tau(\check{f})^{\perp}$ is a maximal ideal in $\mathcal{M}_{c}(G / / K)$, by Theorem [6. In the proof of Theorem 12 we have seen that $\tau(\breve{f})^{\perp}=M_{f}$. The proof of the statement that $M_{f}$ is exponential is included in the proof of Theorem 12 too.

Now we suppose that the ideal $M_{f}$ is an exponential maximal ideal and $f$ is normed. Then we define $\Phi(\mu)=\langle\mu, \breve{f}\rangle$ for each $\mu$ in $\mathcal{M}_{c}(G / / K)$. We can 
perform the same calculation as in the proof of Theorem 12 to show that $\Phi$ is a multiplicative functional of the algebra $\mathcal{M}_{c}(G / / K)$. As

$$
\Phi\left(\delta_{e}^{\#}\right)=\left\langle\delta_{e}, \breve{f}\right\rangle=\check{f}(e)=f(e)=1,
$$

hence $\Phi$ is nonzero. The statement about the kernel of $\Phi$ is proved in Theorem 12, too.

Finally, we suppose that the mapping $\mu \mapsto\langle\mu, \breve{f}\rangle$ is a nonzero multiplicative functional of the algebra $\mathcal{M}_{c}(G / / K)$ with kernel $M_{f}$ and $f$ is normed. We have for each $x$ in $G$

$$
f(x)=\left\langle\delta_{x^{-1}}^{\#}, \check{f}\right\rangle=\Phi\left(\delta_{x^{-1}}^{\#}\right)
$$

hence

$$
\begin{gathered}
f(x) f(y)=\Phi\left(\delta_{x^{-1}}^{\#}\right) \Phi\left(\delta_{y^{-1}}^{\#}\right)=\Phi\left(\delta_{x^{-1}}^{\#} * \delta_{y^{-1}}^{\#}\right)=\left\langle\delta_{x^{-1}}^{\#} * \delta_{y^{-1}}^{\#}, \breve{f}\right\rangle= \\
\int_{G} \int_{G} \check{f}(u v) d \delta_{x^{-1}}^{\#}(u) d \delta_{y^{-1}}^{\#}(v)= \\
\int_{G} \int_{G} \int_{K} \int_{K} \int_{K} \int_{K} \check{f}\left(k u l k^{\prime} v l^{\prime}\right) d \omega(k) d \omega(l) d \omega\left(k^{\prime}\right) d \omega\left(l^{\prime}\right) d \delta_{x^{-1}}(u) d \delta_{y^{-1}}(v)= \\
\int_{G} \int_{G} \int_{K} \check{f}(u l v) d \omega(l) d \delta_{x^{-1}}(u) d \delta_{y^{-1}}(v)= \\
\int_{K} \check{f}\left(x^{-1} l y^{-1}\right) d \omega(l)=\int_{K} f(y l x) d \omega(l),
\end{gathered}
$$

and the theorem is proved.

\section{The case $N=\mathbb{R}^{n}$ and $K=S O(n)$}

We consider the general situation exhibited in Section 5 with $N=\mathbb{R}^{n}$ and $K=S O(n)$, the special orthogonal group. In this case the semidirect product $G=S O(n) \ltimes \mathbb{R}^{n}$ is called the group of Euclidean motions (see also [2]). The elements $(k, a)$ in $G$ can be thought as the product of a rotation $k$ in $S O(n)$ and a translation by $a$ in $\mathbb{R}^{n}$. Hence the pair $g=(k, a)$ operates on $\mathbb{R}^{n}$ by the rule

$$
g \cdot x=k \cdot x+a
$$

for each $x$ in $\mathbb{R}^{n}$. By Corollary 5 , we conclude that $\left(\mathbb{R}^{n}, S O(n)\right)$ is a Gelfand pair. $K$-radial functions are those continuous functions $f: \mathbb{R}^{n} \rightarrow \mathbb{C}$ satisfying

$$
f(x)=f(k \cdot x)
$$

whenever $x$ is in $\mathbb{R}^{n}$ and $k$ is a real orthogonal $n \times n$ matrix with determinant +1 . Similarly, the compactly supported measure $\mu$ is $K$-radial if and only if it satisfies

$$
\int_{\mathbb{R}^{n}} f(x) d \mu(x)=\int_{\mathbb{R}^{n}} f(k \cdot x) d \mu(x)
$$


for each continuous function $f: \mathbb{R}^{n} \rightarrow \mathbb{C}$ and for each real orthogonal $n \times n$ matrix $k$ with determinant +1 .

The proof of the following result can be found in [2].

Theorem 14. The $K$-radial function $\varphi: \mathbb{R}^{n} \rightarrow \mathbb{C}$ is a $K$-spherical function if and only if it is $\mathcal{C}^{\infty}$, it is an eigenfunction of the Laplacian, and $\varphi(0)=1$.

Let $\varphi$ be a $\mathcal{C}^{\infty} K$-radial function on $\mathbb{R}^{n}$, which is a solution of $\Delta \varphi=\lambda \varphi$ for some complex number $\lambda$. Let $\varphi_{0}$ be defined for real $r$ as

$$
\varphi_{0}(r)=\varphi(r, 0,0, \ldots, 0),
$$

then

$$
\varphi(x)=\varphi_{0}(\|x\|)
$$

holds for each $x$ in $\mathbb{R}^{n}$ and $\varphi_{0}$ is a regular even solution of the differential equation

$$
\frac{d^{2} \varphi_{0}}{d r^{2}}+\frac{n-1}{r} \frac{d \varphi_{0}}{d r}=\lambda \varphi_{0}
$$

hence it is proportional to the Bessel-function $J_{\lambda}$ defined by

$$
J_{\lambda}(r)=\Gamma\left(\frac{n}{2}\right) \sum_{k=0}^{\infty} \frac{\lambda^{k}}{k ! \Gamma\left(k+\frac{n}{2}\right)}\left(\frac{r}{2}\right)^{2 k} .
$$

As $J_{\lambda}(0)=1$ follows that $\varphi$ is a $K$-spherical function if and only if

$$
\varphi(x)=J_{\lambda}(\|x\|)
$$

holds for each $x$ in $\mathbb{R}^{n}$ with some complex number $\lambda$ (see [2]).

\section{Spherical monomials}

The function $\varphi$ in $\mathcal{C}(G / / K)$ is called a generalized $K$-spherical monomial, if there exists a $K$-spherical function $s$ and a natural number $n$ such that we have for each $x, y_{1}, y_{2}, \ldots, y_{n+1}$

$$
D_{s ; y_{1}} * D_{s ; y_{2}} * \cdots * D_{s, y_{n+1}} * \varphi(x)=0
$$

Lemma 2. The nonzero function $\varphi$ in $\mathcal{C}(G / / K)$ is a generalized $K$-spherical monomial if and only if there exists a unique exponential maximal ideal in $\mathcal{M}_{c}(G / / K)$ and a natural number $n$ such that

$$
M^{n+1} \subseteq \operatorname{Ann} \tau(\varphi) .
$$

Proof. Let $\varphi \neq 0$ be a generalized $K$-spherical monomial. Then there exists a $K$-spherical function $s$ and a natural number $n$ such that (3) holds for each $x, y_{1}, y_{2}, \ldots, y_{n+1}$ in $G$, and, by the commutativity of $\mathcal{M}_{c}(G / / K)$, we have

$$
D_{s ; y_{1}} * D_{s ; y_{2}} * \cdots * D_{s, y_{n+1}} * \psi(x)=0
$$


whenever $\psi$ is in $\tau(\varphi)$. As the measures $D_{s ; y_{1}} * D_{s ; y_{2}} * \cdots * D_{s, y_{n+1}}$ generate the ideal whose closure is $M_{s}^{n+1}$ we infer that $M_{s}^{n+1} \subseteq \operatorname{Ann} \tau(\varphi)$. As $\varphi$ is nonzero, there exists a maximal ideal $M$ in $\mathcal{M}_{c}(G / / K)$ such that $\operatorname{Ann} \tau(\varphi) \subseteq M$, which implies

$$
M_{s}^{n+1} \subseteq M
$$

Maximal ideals are prime, hence we conclude $M=M_{s}$, which is an exponential maximal ideal. If $N$ is a maximal $K$-ideal with the property that

$$
N^{k+1} \subseteq \operatorname{Ann} \tau(\varphi)
$$

for some natural number $n$, then we have

$$
N^{k+1} \subseteq M_{s},
$$

and $M_{s}$ is prime, hence we conclude $N=M_{s}$.

The converse statement is obvious.

If $s$ is a $K$-spherical function and $M_{s}^{n+1} \subseteq \operatorname{Ann} \tau(\varphi) \subseteq M_{s}$ holds for some natural number $n$, then we say that the generalized $K$-spherical monomial $\varphi$ corresponds to $s$. By the above lemma, $s$ is unique. The smallest natural number $n$ with this property is called the degree of $\varphi$. The zero function is a generalized $K$-spherical monomial but we do not assign a degree to it.

We call a generalized $K$-spherical monomial simply a $K$-spherical monomial, if it generates a finite dimensional $K$-variety. By the definition, the set of all generalized $K$-spherical monomials of degree $n$ corresponding to the $K$-spherical function $s$ is $\operatorname{Ann} M_{s}^{n+1}$ and the set of all generalized $K$-spherical monomials corresponding to $s$ is $\bigcup_{n \in \mathbb{N}}$ Ann $M_{s}^{n+1}$. Further, given the $K$-variety $V$ the set of all generalized $K$-spherical monomials of degree $n$ corresponding to the $K$-spherical function $s$ in $V$ is $V \cap A n n M_{s}^{n+1}$ and the set of all generalized $K$-spherical monomials corresponding to $s$ in $V$ is $V \cap \bigcup_{n \in \mathbb{N}} \operatorname{Ann} M_{s}^{n+1}$.

\section{Spherical spectral analysis and spectral synthesis}

Let $V$ be a $K$-variety. We say that $K$-spectral analysis holds for $V$, if in every nonzero sub- $K$-variety of $V$ there is a $K$-spherical function. If $G$ is commutative, then this is equivalent to spectral analysis for the variety $V$ in $\mathcal{C}(G / K)$.

Theorem 15. Let $V$ be a $K$-variety. $K$-spectral analysis holds for $V$ if and only if every maximal ideal in $\mathcal{M}_{c}(G / / K)$ containing $A n n V$ is exponential. In other words, $K$-spectral analysis holds for $V$ if and only if every maximal ideal in $\mathcal{M}_{c}(G / / K) / \operatorname{Ann} V$ is exponential.

Proof. If $K$-spectral analysis holds for $V$ and $M$ is a maximal $K$-ideal with Ann $V \subseteq M$, then obviously $V=\operatorname{Ann} \operatorname{Ann} V \supseteq \operatorname{Ann} M$. Hence Ann $M$ is a nonzero sub- $K$-variety of $V$, which includes a $K$-spherical function $s$. It follows 
that $\operatorname{Ann} \tau(s)$, which is a maximal $K$-ideal, is a superset of $M$, hence they are equal: Ann $\tau(s)=M$. As Ann $\tau(s)$ is exponential, the necessity part of theorem is proved.

Suppose now that every maximal $K$-ideal containing $\operatorname{Ann} V$ is exponential and let $W$ be a nonzero sub- $K$-variety in $V$. Then Ann $W \supseteq$ Ann $V$, hence every maximal $K$-ideal containing Ann $W$ also contains Ann $V$, thus it is exponential. Let $M$ be one of them; then we have $M=M_{s}$ for some $K$-spherical function $s$, further Ann $W \supseteq M_{s}=\operatorname{Ann} \tau(s)$. We conclude $\tau(s) \subseteq W$, and the theorem is proved.

This is the analogue of the spectral analysis theorems Theorem 14.2 and Theorem 14.3 on p. 203 in 8 .

We say that $K$-spectral analysis holds on $G$, if $K$-spectral analysis holds for each $K$-variety on $G$. This means that $K$-spectral analysis holds for $\mathcal{C}(G / / K)$. If $G$ is commutative, then this is exactly spectral analysis on the group $G / K$.

Corollary 6. $K$-spectral analysis holds on $G$ if and only if every maximal ideal in $\mathcal{M}_{c}(G / / K)$ is exponential.

For instance, if $G$ is a discrete Abelian group and $K$ is any finite subgroup, then $\mathcal{M}_{c}(G / / K)$ is isomorphic to $\mathcal{M}_{c}(G / K)$. In this case the condition of the theorem is satisfied if and only if the torsion-free rank of the group $G / K$ is less than the continuum (see e.g. [5]).

Let $V$ be a $K$-variety. We say that $V$ is $K$-synthesizable, if the $K$-spherical monomials span a dense subspace in $V$. We say that $K$-spectral synthesis holds for $V$, if every sub- $K$-variety of $V$ is $K$-synthesizable. We say that $K$-spectral synthesis holds on $G$, if every $K$-variety on $G$ is $K$-synthesizable. It is easy to see, that $K$-spectral synthesis implies $K$-spectral analysis for a variety. Clearly, $K$ synthesizability and $K$-spectral synthesis reduce to synthesizability and spectral synthesis on $G / K$ if $G$ is commutative. If, for instance, $G$ is a discrete Abelian group, and $K$ is a finite subgroup, then $K$-spectral synthesis holds on $G$ if and only if the torsion-free rank of $G / K$ is finite (see [6]).

For synthesizability of varieties we have the following result (see [8, 9, 10]).

Theorem 16. The nonzero $K$-variety $V$ is $K$-synthesizable if and only if

$$
\text { Ann } V=\bigcap_{M} \bigcap_{n \in \mathbb{N}}\left(\operatorname{Ann} V+M^{n+1}\right),
$$

where the first intersection is taken for all exponential maximal ideals $M$ containing Ann $V$ and $\mathcal{M}_{c}(G / / K) / M^{n+1}$ is finite dimensional.

Proof. By definition of synthesizability and by the remarks at the end of the previous section, the $K$-variety $V$ is synthesizable if and only if

$$
V=\sum_{M} \sum_{n \in \mathbb{N}}\left(V \cap \operatorname{Ann} M^{n+1}\right) .
$$


On the other hand, applying Theorem 8. on p. 6 in [10], our statement follows.

In the case if $N$ is a locally compact Abelian group and $K$ is a compact group of its automorphisms as it has been discussed in Section 5 instead of " $K$ spectral analysis on $G$ ", resp. " $K$-spectral synthesis on $G$ " we can say simply " $K$-spectral analysis on $N$ ", resp. " $K$-spectral synthesis on $N$ ".

\section{Extension of L. Schwartz's spectral synthesis}

In his monumental and pioneer work [7 L. Schwartz proved the following fundamental result.

Theorem 17. (L. Schwartz) Spectral synthesis holds on the reals. In other words, in every linear and translation invariant space of continuous complex valued functions on the real line, which is closed with respect to uniform convergence on compact sets all functions of the form $x \mapsto x^{n} e^{\lambda x}$ span a dense subspace ( $n$ is a natural number, $\lambda$ is a complex number).

As a consequence, every complex valued continuous function on the real line can be uniformly approximated on compact sets by linear combinations of functions of the above form, the exponential monomials, which are uniform limits on compact sets of linear combinations of translates of the given function. This statement clearly includes the deep existence theorem on spectral analysis: given any nonzero continuous complex valued function on the reals the smallest linear space including the function and is closed under translation and uniform convergence on compact sets contains nonzero exponential monomials. The proof of this beautiful result depends on hard complex function theory. Since the above result was published a great number of efforts have been made to extend it to several variables, that is, to varieties in $\mathbb{R}^{n}$, but an extension was possible only in the case of some special varieties, no general result was available. Finally, almost thirty years later D. I. Gurevich provided counterexamples for the corresponding result in $\mathbb{R}^{2}$. In fact, in 3 , he gave an example for a variety in $\mathbb{R}^{2}$ whose annihilator is generated by two measures and no spectral analysis holds on it, and for another variety whose annihilator is generated by three measures and no spectral synthesis holds on it. These negative results verify the conjecture that a direct generalization to translation invariant closed subspaces in $\mathbb{R}^{n}$ may not be proper way to extend Schwartz's result. In fact, here we show that a more sophisticated, but still natural generalization is possible in terms of spherical functions. Our basic observation is the following: instead of spectral synthesis we consider $K$-spectral synthesis on $\mathbb{R}^{n}$, where $K=S O(n)$, the special orthogonal group acting on $\mathbb{R}^{n}$ as it was discussed in Section 7 In the special case $n=1$ we have $K=S O(1)=\{i d\}$, hence in this case $K$ spectral synthesis reduces to ordinary spectral synthesis. However, if $n>1$, then $K=S O(n)$ is non-trivial, and $K$-spectral synthesis is essentially different from ordinary spectral synthesis. Hence our forthcoming result shows that a 
successful generalization of L. Schwartz's result in this direction is possible if "translation invariance" is replaced by "invariance with respect to Euclidean motions". To prove this statement we shall need some preliminary results. In what follows we let $K=S O(n)$, the special orthogonal group.

For each $\mu$ in $\mathcal{M}_{c}(\mathbb{R})$ and $f$ in $\mathcal{C}_{K}\left(\mathbb{R}^{n}\right)$ we define $\mu_{K}$ by the equation

$$
\left\langle\mu_{K}, f\right\rangle=\left\langle\mu, f_{0}\right\rangle,
$$

where the function $f_{0}: \mathbb{R} \rightarrow \mathbb{C}$ is given by

$$
f_{0}(r)=f(r, 0,0, \ldots, 0)
$$

for each $r$ in $\mathbb{R}$. As $f$ is $K$-radial, and $K=S O(n)$ acts transitively on the unit sphere in $\mathbb{R}^{n}$ we have that $\|x\|=\|y\|$ implies $f(x)=f(y)$ for $x, y$ in $\mathbb{R}^{n}$. In particular, $f(\|x\|, 0,0, \ldots, 0)=f(x)$, and

$$
f(x)=f_{0}(\|x\|)
$$

holds for each $x$ in $\mathbb{R}^{n}$.

Theorem 18. The mapping $\mu \mapsto \mu_{K}$ is a continuous algebra homomorphism of $\mathcal{M}_{c}(\mathbb{R})$ onto $\mathcal{M}_{K}\left(\mathbb{R}^{n}\right)$.

Proof. It is easy to see that $\mu_{K}$ is a compactly supported measure on $\mathbb{R}^{n}$, hence it is a linear functional on $\mathcal{C}_{K}\left(\mathbb{R}^{n}\right)$. Further, we have for each $\mu, \nu$ in $\mathcal{M}_{c}(\mathbb{R})$ and $f$ in $\mathcal{C}_{K}\left(\mathbb{R}^{n}\right)$

$$
\begin{gathered}
\left\langle(\mu * \nu)_{K}, f\right\rangle=\left\langle\mu * \nu, f_{0}\right\rangle=\int_{\mathbb{R}} \int_{\mathbb{R}} f_{0}(s+r) d \mu(s) d \nu(r)= \\
\int_{\mathbb{R}} \int_{\mathbb{R}} f(s+r, 0,0, \ldots, 0) d \mu(s) d \nu(r),
\end{gathered}
$$

and

$$
\begin{gathered}
\left\langle\mu_{K} * \nu_{K}, f\right\rangle=\int_{\mathbb{R}^{n}} \int_{\mathbb{R}^{n}} f(x+y) d \mu_{K}(x) d \nu_{K}(y)= \\
\int_{\mathbb{R}^{n}}\left[\int_{\mathbb{R}} f\left(s+y_{1}, y_{2}, \ldots, y_{n}\right) d \mu(s)\right] d \nu_{K}(y)= \\
\int_{\mathbb{R}}\left[\int_{\mathbb{R}^{n}} f\left(s+y_{1}, y_{2}, \ldots, y_{n}\right) d \nu_{K}(y)\right] d \mu(s)=\int_{\mathbb{R}} \int_{\mathbb{R}} f(s+r, 0,0, \ldots, 0) d \nu(r) d \mu(s),
\end{gathered}
$$

hence $(\mu * \nu)_{K}=\mu_{K} * \nu_{K}$, and $\mu \mapsto \mu_{K}$ is an algebra homomorphism. Finally, for each $\varphi$ in $\mathcal{M}_{c}(\mathbb{R})$ and $x$ in $\mathbb{R}^{n}$ we define

$$
f_{\varphi}(x)=\varphi(\|x\|) .
$$

Then $f_{\varphi}$ is in $\mathcal{C}_{K}\left(\mathbb{R}^{n}\right)$. Given $\xi$ in $\mathcal{M}_{K}\left(\mathbb{R}^{n}\right)$ we let

$$
\langle\mu, \varphi\rangle=\left\langle\xi, f_{\varphi}\right\rangle .
$$


We then have for each $f$ in $\mathcal{C}_{K}\left(\mathbb{R}^{n}\right)$

$$
\begin{gathered}
\left\langle\mu_{K}, f\right\rangle=\left\langle\mu, f_{0}\right\rangle=\left\langle\xi, f_{f_{0}}\right\rangle=\int_{\mathbb{R}^{n}} f_{f_{0}}(x) d \xi(x)=\int_{\mathbb{R}^{n}} f_{0}(\|x\|) d \xi(x)= \\
\int_{\mathbb{R}^{n}} f(\|x\|, 0,0, \ldots, 0) d \xi(x)=\int_{\mathbb{R}^{n}} f(x) d \xi(x)=\langle\xi, f\rangle,
\end{gathered}
$$

which means $\mu_{K}=\xi$, hence the mapping $\mu \mapsto \mu_{K}$ is surjective. Its continuity is obvious.

By Theorem [16] the $K$-synthesizability of a $K$-variety can be expressed purely in terms of the annihilator of the $K$-variety. We introduce the following terminology: let $R$ be a commutative complex topological algebra with unit. The proper closed ideal $I$ in $R$ is called synthesizable if

$$
I=\bigcap_{M} \bigcap_{n \in \mathbb{N}}\left(I+M^{n+1}\right),
$$

where the first intersection is taken for all exponential maximal ideals $M$ containing $I$ and $R / M^{n+1}$ is finite dimensional. Accordingly, we say that spectral synthesis holds on $R$, if every closed ideal $I$ in $R$ satisfies the above equation. In particular, $K$-spectral synthesis holds on $G$ if and only if this equation holds for each proper closed ideal $I$ in $\mathcal{M}_{c}(G / / K)$. The following theorem is a simple consequence.

Theorem 19. Let $R, Q$ be commutative complex topological algebras with unit. If spectral synthesis holds on $R$, and there exists a continuous surjective homomorphism $\Phi: R \rightarrow Q$, then spectral synthesis holds on $Q$.

Proof. Let $M$ be a maximal ideal with $Q$, then $M=\Phi(N)$ with some ideal $N$ in $R$ such that $N=\Phi^{-1}(M)$. Let $\psi: Q \rightarrow Q / M$ denote the natural mapping, then $\psi$ is continuous and open. We define

$$
F(r)=\psi(\Phi(r))
$$

for each $r$ in $R$, then $F: R \rightarrow Q / M$ is a continuous homomorphism. Clearly, $F$ is surjective. If $F(r)=0$, then $\Phi(r)$ is in $\operatorname{Ker} \psi=M$, that is, $r$ is in $N$. It follows that $R / N \cong Q / M$, a field, hence $N=\operatorname{Ker} F$ is a closed maximal ideal. By assumption, $N$ is exponential, hence $M$ is exponential, too.

Let $J$ be a proper closed ideal in $Q$ and let $I=\Phi^{-1}(J)$. Then $I$ is a proper closed ideal in $R$, hence it is synthesizable, by assumption. It follows that (4) holds. Then we have

$$
J=\bigcap_{\Phi(M)} \bigcap_{n \in \mathbb{N}}\left(J+\Phi(M)^{n+1}\right),
$$

and here the first intersection extends for all maximal ideals $\Phi(M)$ containing $J$. Indeed, the left hand side is clearly a subset of the right hand side. Suppose 
now that $q=\Phi(r)$ is not in $J$, then $r$ is not in $I$. By equation (4), there exists a maximal ideal $M$ with $I \subseteq M$, and a natural number $n_{0}$ such that $r$ is not in $I+M^{n_{0}+1}$, hence $q=\Phi(r)$ is not in $J+\Phi(M)^{n_{0}+1}$. It follows that (5) holds.

What is left is to show that $Q / \Phi(M)^{n+1}$ is finite dimensional for every maximal ideal $M$ with $I \subseteq M$ and for each natural number $n$. We define $F: R / M^{n+1} \rightarrow Q / \Phi(M)^{n+1}$ by

$$
F\left(r+M^{n+1}\right)=\Phi(r)+\Phi(M)^{n+1}
$$

for each $r$ in $R$. We have to show that the value of $F$ is independent of the choice of $r$ in the coset $r+M^{n+1}$. Suppose that $r-r_{1}$ is in $M^{n+1}$, that is $r-r_{1}=\sum x_{1} x_{2} \cdots x_{n+1}$, where the sum is finite, and $x_{1}, x_{2}, \ldots, x_{n+1}$ is in $M$. Then

$$
\Phi(r)=\Phi\left(r_{1}\right)+\sum \Phi\left(x_{1}\right) \Phi\left(x_{2}\right) \cdots \Phi\left(x_{n+1}\right),
$$

hence $\Phi(r)$ and $\Phi\left(r_{1}\right)$ are in the same coset of $\Phi(M)^{n+1}$. As $F$ is clearly a surjective homomorphism, we infer that $Q / \Phi(M)^{n+1}$ is finite dimensional and the proof is complete.

Now we are ready to present our main result on the extension of L. Schwartz's spectral synthesis result Theorem 17.

Theorem 20. Let $K=S O(n)$ for every positive integer $n$ acting on $\mathbb{R}^{n}$. Then $K$-spectral synthesis holds on $\mathbb{R}^{n}$.

Proof. Our statement is a consequence of L. Schwartz's Theorem 17 using Theorem 18, and Theorem 19,

\section{References}

[1] J. Dieudonné, Treatise on analysis. Vol. VI, Academic Press, Inc., New York-London, 1978.

[2] G. van Dijk, Introduction to harmonic analysis and generalized Gelfand pairs. Walter de Gruyter \& Co., Berlin, 2009.

[3] D. I. Gurevič, Counterexamples to a problem of L. Schwartz, Funkcional. Anal. i Priložen. 9(2), (1975), 29-35.

[4] J. L. Kelley and I. Namioka, Linear topological spaces, Springer Verlag, New York, 1976.

[5] M. Laczkovich and G. Székelyhidi, Harmonic analysis on discrete Abelian groups, Proc. Amer. Math. Soc. 133(6), (2005), 1581-1586.

[6] M. Laczkovich and L. Székelyhidi, Spectral synthesis on discrete Abelian groups, Math. Proc. Camb. Phil. Soc. 143(1), (2007), 103-120. 
[7] L. Schwartz, Théorie générale des fonctions moyenne-périodiques, Ann. of Math. 48(4), (1947), 857-929.

[8] L. Székelyhidi, Harmonic and spectral analysis. World Scientific Publishing Co. Pte. Ltd., Hackensack, NJ, 2014.

[9] L. Székelyhidi, Annihilator methods in discrete spectral synthesis, Acta Math. Hung., 143(2), (2014), 351-366.

[10] L. Székelyhidi, Annihilator methods for spectral synthesis on locally compact Abelian groups, Monatsh. Math., 180(2), (2016), 357-371. 